\title{
Effects of mavacamten on Ca2+-sensitivity of contraction as sarcomere length varied in human myocardium
}

\author{
Peter Awinda ${ }^{1}$, Yemeserach Bishaw ${ }^{1}$, Marissa Watanabe ${ }^{1}$, Maya Guglin ${ }^{2}$, Kenneth \\ Campbell ${ }^{2}$, and Bertrand Tanner ${ }^{1}$ \\ ${ }^{1}$ Washington State University \\ ${ }^{2}$ University of Kentucky
}

May 6, 2020

\begin{abstract}
Background and Purpose: Heart failure can reflect impaired contractile function at the myofilament level. In healthy hearts, myofilaments become more sensitive to $\mathrm{Ca} 2+$ as cells are stretched. This represents a fundamental property of myocardium that contributes to the Frank-Starling response, although the molecular mechanisms underlying the effect remain unclear. Mavacamten is a drug that binds to myosin, which is under investigation as a potential therapy for cardiovascular disease. We tested how mavacamten affects the sarcomere-length dependence of Ca2+-sensitive isometric contraction to determine how mavacamten might modulate the Frank-Starling mechanism. Experimental Approach: Multicellular preparations from the left ventricular free wall of hearts procured from organ donors were chemically permeabilized and Ca2+-activated in the presence or absence of $0.5 \mu \mathrm{M}$ mavacamten at 1.9 or $2.3 \mu \mathrm{m}$ sarcomere length $\left(37^{\circ} \mathrm{C}\right)$. Isometric force and frequencydependent viscoelastic myocardial stiffness measurements were made. Key Results: At both sarcomere lengths, mavacamten reduced maximal force and $\mathrm{Ca} 2+$-sensitivity of contraction. In the presence and absence of mavacamten, Ca2+-sensitivity of force increased as sarcomere length increased. This suggests that the length-dependent activation response was maintained in human myocardium, even though mavacamten reduced Ca2+-sensitivity. There were subtle effects of mavacamten reducing force values under relaxed conditions ( $\mathrm{pCa}$ 8.0), as well as slowing myosin cross-bridge recruitment and speeding cross-bridge detachment under maximally activated conditions (pCa 4.5). Conclusion and Implications: Mavacamten did not eliminate sarcomere lengthdependent increases in the Ca2+-sensitivity of contraction in myocardial strips from organ donors at physiological temperature. Pharmaceuticals that modulate myofilament function may be useful therapies for cardiovascular disease.
\end{abstract}

\section{Introduction}

Cardiovascular disease remains a leading cause of death world-wide. Heart failure occurs when a patient's heart loses the capacity to adequately pump blood around their body (Mozaffarian et al., 2016). Heart failure is a growing health problem affecting $\sim 30$ million people worldwide, with $50 \%$ of heart failure patients dying within five years of diagnosis (Ambrosy et al., 2014; Mozaffarian et al., 2016). The causes of heart-failure are multi-factorial, but dysregulated myofilament function within the sarcomere is a leading contributor. Recently, multiple pharmaceutical compounds have been developed to directly influence myofilament protein function as potential new therapies for cardiac disease (Cleland et al., 2011; Malik et al., 2011; Green et al., 2016; Teerlink et al., 2016; Kawas et al., 2017; Grillo et al., 2018; Heitner et al., 2019).

Cardiac muscle contraction is powered by cyclic interactions between myosin cross-bridges along thickfilaments and actin binding sites along thin-filaments (Huxley and Hanson, 1954; Lymn and Taylor, 1971). Contractility is modulated by several well-known mechanisms including: i) $\mathrm{Ca}^{2+}$-regulation via the thin filament proteins troponin and tropomyosin, which modulates the number of available actin binding on a thin-filament as intracellular $\left[\mathrm{Ca}^{2+}\right]$ rises and falls throughout a heartbeat; and ii) thick-to-thin filament 
overlap, which determines how many cross-bridges are close enough to bind with $\mathrm{Ca}^{2+}$-activated actin sites. A mechanosensitive thick-filament regulatory mechanism has been discovered in the last few years as well, whereby myosin heads transition between OFF (also called the super-relaxed state, or interacting heads motif) and ON states (also called the disordered relaxed state) (Hooijman et al., 2011; Campbell, 2017). Heads in the OFF state are unable to bind actin (Fig. 1), while those in the ON state can form cross-bridges by attaching to actin (Spudich, 2015; Anderson et al., 2018; Liu et al., 2018). OFF-ON transitions can be very dynamic (Fusi et al., 2017; Reconditi et al., 2017; Piazzesi et al., 2018) and equilibrium kinetics are known to be regulated by i) biochemical and steric interactions with thick-filament regulatory proteins [regulatory light chain (Kampourakis et al., 2016; Zhang et al., 2017) and cardiac myosin binding protein-C (McNamara et al., 2015, 2017)], as well as ii) myocardial force levels (Linari et al., 2015; Ait-Mou et al., 2016; Fusi et al., 2016; Kampourakis et al., 2016; Campbell et al., 2018). These multiple regulation pathways combine to influence length-dependent activation of contraction, wherein the myofilaments become more sensitive to $\mathrm{Ca}^{2+}$ as muscle cells are stretched. Length-dependent activation is an important cellular-level mechanism that underpins the Frank-Starling mechanism and enables the heart to increase cardiac output in response to elevated filling pressures.

Mavacamten (formerly known as MYK-461; MyoKardia Inc.) is a pharmaceutical under investigation to treat cardiac hypercontractility, a phenotype commonly associated with a form heart disease called hypertrophic cardiomyopathy (HCM) (Green et al., 2016; Stern et al., 2016). HCM affects ${ }^{1}$ in 300 people, and typically causes thickening and stiffening of the ventricular wall, thereby impairing ventricular filling and reducing cardiac output (Klein et al., 1965; Brandt et al., 1967; Wilson et al., 1967; Stewart et al., 1968; Maron et al., 1995; Semsarian et al., 2015). Mavacamten binds to myosin, inhibits actin-myosin ATPase activity, and stabilizes the myosin OFF state (Green et al., 2016; Anderson et al., 2018; Rohde et al., 2018; Toepfer et al., 2019b). Solution biochemistry, in vitro motility, and single molecule assays have shown that mavacamten slows the rates of inorganic phosphate (Pi) and ADP release (Green et al., 2016; Kawas et al., 2017; Rohde et al., 2018). Mavacamten also slows the rate of cross-bridge recruitment in skinned rodent myocardium (Mamidi et al., 2018). Increases in mavacamten concentration reduced sarcomere shortening, while increasing the speed of relaxation in isolated electrically paced myocytes (Toepfer et al., 2019b, 2019a). Although there are mixed findings with respect to mavacamten either reducing or not affecting $\mathrm{Ca}^{2+}$-sensitivity of contraction in skinned myocardial strips (Green et al., 2016; Mamidi et al., 2018), studies have shown that mavacamten consistently reduces maximal force production (Green et al., 2016; Anderson et al., 2018; Mamidi et al., 2018).

These combined effects of mavacamten on myosin force production and ATPase activity are starting to provide evidence that mavacamten may reduce hypercontractility at the level of the myosin motor (Heitner et al., 2019; Tuohy et al., 2020). However, prior studies have not provided a consistent interpretation by which mavacamten affects myosin cross-bridge kinetics, nor how mavacamten might influence length-dependent myocardial function (i.e. the cellular basis of the Frank-Starling law). Therefore, we tested the effect of mavacamten on $\mathrm{Ca}^{2+}$-activated force production at 1.9 and $2.3 \mu \mathrm{m}$ sarcomere length in permeabilized myocardial strips from organ donors.

\section{Methods}

\section{Human tissue samples}

Cardiac samples were obtained from 6 organ donors (2 male) at the University of Kentucky (Table 1). Their mean age was 45.5 (range 10-61) years. As previously described in detail (Blair et al., 2016), hearts were passed to a researcher as soon as they were excised from the body, immediately placed in ice cold saline slush and transported back to the laboratory where tissue samples ( $\sim 500 \mathrm{mg})$ were snap frozen in liquid nitrogen and stored at $-150^{\circ} \mathrm{C}$ within $\sim 20$ minutes. Mid-myocardial sections of the left ventricular free wall were shipped overnight on dry ice to Washington State University and stored at $-80^{\circ} \mathrm{C}$ for $1-3$ weeks, until they were dissected for mechanics and biochemical experiments. All procedures were approved by the University of Kentucky Institutional Review Board. 


\section{Solutions}

Muscle mechanics solution concentrations were formulated by solving equations describing ionic equilibria (Godt and Lindley, 1982) and all concentrations are listed in mM unless otherwise noted. Skinning solution: $50 \mathrm{BES}, 30.83 \mathrm{~K}$ propionate, $10 \mathrm{Na}$ azide, $20 \mathrm{EGTA}, 6.29 \mathrm{MgCl}_{2}$, 6.09 ATP, 1 DTT, 20 BDM, 50 Leupeptin, 275 Pefabloc, and 1 E-64; with 1\% Triton-X100 (wt/vol) and 50\% glycerol (wt/vol). Storage solution: same as skinning solution without Triton-X100. Relaxing solution: pCa 8.0 (pCa $\left.=-\log _{10}\left[\mathrm{Ca}^{2+}\right]\right), 5$ EGTA, 5 MgATP, $1 \mathrm{Mg}^{2+}, 0.3 \mathrm{P}_{\mathrm{i}}, 35$ phosphocreatine, $300 \mathrm{U} / \mathrm{mL}$ creatine kinase, $\mathrm{pH} 7.0$, at 200 ionic strength adjusted with $\mathrm{Na}$ methanesulfonate. Activating solution: same as relaxing solution, but with pCa 4.5.

Mavacamten (MYK-461) was purchased from Axon Medichem (Reston, VA, USA) and dissolved in dimethylsulfoxide (DMSO) to give a $1 \mathrm{mM}$ stock solution. This was then mixed with relaxing and activating solutions to yield experimental solutions containing $0.5 \mu \mathrm{M}$ mavacamten and $0.05 \%$ DMSO (vol/vol). The $0.5 \mu \mathrm{M}$ mavacamten concentration was chosen as an intermediate between the $\mathrm{IC}_{50}$ value of $0.3 \mu \mathrm{M}$ for inhibiting myosin ATPase activity in biochemical assays using murine and bovine myosin (though isometric force appeared less sensitive to mavacmaten than ATPase (Green et al., 2016) ) and plasma levels that effectively relieved left ventricular outflow tract obstruction in clinical trial patients $(350-695 \mathrm{ng} / \mathrm{ml}=1.28-2.54 \mu \mathrm{M}$; (Heitner et al., 2019)).

\section{Mechanical measurements using permeabilized myocardial strips}

Frozen tissue sections were thawed in ice-cold skinning solution and dissected into thin strips $(\sim 180 \mu \mathrm{m}$ in diameter and $700 \mu \mathrm{m}$ long). These were skinned overnight at $4^{\circ} \mathrm{C}$, transferred to storage solution, and stored at $-20^{\circ} \mathrm{C}$ for 0-5 days. On the day of experiments, strips were mounted between a motor (P841.40, Physik Instrumente, Auburn, MA) and a strain gauge (AE801, Kronex, Walnut Creek, CA), lowered into a $30 \mu \mathrm{L}$ droplet of relaxing solution (with or without mavacamten) and stretched to a sarcomere length of 1.9 or $2.3 \mu \mathrm{m}$ as measured by digital Fourier transform analysis (IonOptix Corp, Milton, MA). Solutions were maintained at physiological temperature $\left(37^{\circ} \mathrm{C}\right)$ throughout each experiment, using 2-3 strips at each condition for each heart.

Strips were activated in solutions (with or without mavacamten) at pCa values ranging from 8.0 to 4.5 to measure the steady-state, isometric force-pCa relationship. Force values were normalized to the crosssectional area of each preparation and reported as stress values with units of $\mathrm{kN} \mathrm{m}^{-2}$. Stress-pCa curves from each strip were fit to a 4-parameter Hill equation using MATAB (version 9.0.4, Mathworks, Natick, MA):

\section{Hosted file}

image1.wmf available at https://authorea.com/users/318807/articles/448686-effects-ofmavacamten-on-ca2-sensitivity-of-contraction-as-sarcomere-length-varied-in-humanmyocardium

, Eq. (1)

where $\mathrm{F}_{\text {pas }}$ corresponds to passive stress under relaxed conditions; $\mathrm{F}_{\text {act }}$ corresponds to maximal $\mathrm{Ca}^{2+}{ }_{-}$ activated stress; $\mathrm{pCa}_{50}$ represents the free $\mathrm{Ca}^{2+}$ concentration required to develop half the maximum $\mathrm{Ca}^{2+}$ activated stress, and $\mathrm{n}_{\mathrm{H}}$ is the Hill coefficient.

Sinusoidal length-perturbations of $0.125 \%$ myocardial strip length (clip-to-clip) were applied at 41 discreet frequencies from $0.125-100 \mathrm{~Hz}$ to measure the complex modulus as a function of angular frequency (Kawai and Brandt, 1980; Mulieri et al., 2002; Palmer et al., 2007). The complex modulus represents viscoelastic myocardial stiffness, which arises from the change in stress divided by the change in muscle length that is in-phase (elastic modulus) and out-of-phase (viscous modulus) with the oscillatory length change at each frequency.

Characteristics of the elastic and viscous moduli responses over the measured frequency range provide a signature of cross-bridge binding and cycling kinetics. Shifts in the elastic modulus are useful for assess- 
ing changes in the number of bound cross-bridges between experimental conditions. Shifts in the viscous modulus are useful for assessing changes in the work-producing and work-absorbing characteristics of the myocardium that arise from force-generating cross-bridges. Frequencies producing negative viscous moduli represent regions of work-producing muscle function. The "dip frequency" or frequency of the minimum viscous modulus describes force-generating events and cross-bridge recruitment rate (Mulieri et al., 2002; Campbell et al., 2004). Frequencies producing positive viscous moduli represent regions of work-absorbing muscle function. The "peak frequency" or frequency of the maximum viscous modulus describes cross-bridge distortion events and cross-bridge detachment rate (Campbell et al., 2004; Palmer et al., 2007, 2011). These characteristic regions of minima and maxima in the viscous modulus vs. frequency relationship were used to assess effects of mavacamten on cross-bridge kinetics under maximal $\mathrm{Ca}^{2+}$-activated conditions. Given that viscous moduli were only measured at discrete frequencies, these regions of minima viscous modulus (using $0.125-4 \mathrm{~Hz}$ data) and maxima viscous modulus (using $3-40 \mathrm{~Hz}$ data) were fit to a polynomial using MATLAB to create fitted curves at $0.05 \mathrm{~Hz}$ resolution. From these interpolated curves we extracted the frequency of minimum viscous modulus and frequency of maximum viscous modulus.

\section{Statistical analysis}

Experimental data were analyzed in SAS (version 9.4.3, SAS Institute, Cary, NC) using linear mixed effects models incorporating 2 main effects (mavacamten and sarcomere length) and their interaction for the stresspCa fit parameters from Eq. 1, and frequency parameters extracted from curve-fits to the minima and maxima viscous modulus. These statistical analyses link data from the same hearts to optimize statistical power, and can be considered as the two-way equivalent of a paired t-test (Haynes et al., 2014). Compound symmetry was assumed for the covariance structure and post-hoc analyses were performed using TukeyKramer corrections. P values less than 0.05 were considered significant.

\section{Results}

Effects of mavacamten on $\mathrm{Ca}^{2+}$-activated isometric contraction

Steady-state isometric stress (force normalized to cross-sectional area) was measured as activating $\left[\mathrm{Ca}^{2+}\right]$ increased from pCa 8 (relaxed) to 4.5 (maximally activated) in myocardial strips isolated from donor hearts at 1.9 or $2.3 \mu \mathrm{m}$ sarcomere length (Fig. 2). For both sarcomere lengths, mavacamten decreased $\mathrm{Ca}^{2+}$-activated force and reduced $\mathrm{Ca}^{2+}$-sensitivity of the force-pCa relationship. As sarcomere length increased, $\mathrm{Ca}^{2+}$ activated force and $\mathrm{Ca}^{2+}$-sensitivity of the force-pCa relationship increased for control and mavacamtentreated myocardial strips. Therefore, the typical length-dependent activation response was maintained in the presence of mavacamten. Parameter values for 4-parameter Hill fits (Eq. 1) to each stress-pCa relationship are summarized in Fig. $3\left(\mathrm{~F}_{\text {act }}\right.$ and $\left.\mathrm{F}_{\text {pas }}\right)$ and Fig. $4\left(\mathrm{pCa}_{50}\right.$ and $\left.\mathrm{n}_{\mathrm{H}}\right)$.

At 1.9 and $2.3 \mu \mathrm{m}$ sarcomere length, $0.5 \mu \mathrm{M}$ mavacamten reduced $\mathrm{Ca}^{2+}$-activated force by ${ }^{\sim} 25-30 \%$, compared to control strips $(\mathrm{p}<0.001$ for main effect of mavacamten; Fig. $3 \mathrm{~A}) . \mathrm{Ca}^{2+}$-activated force also increased as sarcomere length increased ( $\mathrm{p}=0.005$ for main effect of length). Similar findings occurred for passive force, with $0.5 \mu \mathrm{M}$ mavacamten reducing passive force at both sarcomere lengths $(\mathrm{p}<0.001$ for main effect of mavacamten; Fig. 3B) and passive force increasing as sarcomere length increased $(\mathrm{p}<0.001$ for main effect of length). These mavacamten-dependent decreases in passive force suggest that a portion of the passive force measured at pCa 8.0 arises from bound cross-bridges, and that mavacamten stabilization of the myosin OFF state reduces this myosin-based contribution to passive force.

$\mathrm{pCa}_{50}$ values of the force-pCa relationship represent the $\mathrm{Ca}^{2+}$ concentration required to produce half-maximal $\mathrm{Ca}^{2+}$-activated force and describe $\mathrm{Ca}^{2+}$-sensitivity of myocardial force development (Fig. 4A). For both sarcomere lengths, $0.5 \mu \mathrm{M}$ mavacamten reduced the $\mathrm{Ca}^{2+}$-sensitivity of force by more than $0.1 \mathrm{pCa}$ units compared to control $\left(\mathrm{p}<0.001\right.$ for main effect of mavacamten; average $\Delta \mathrm{pCa} \mathrm{a}_{50}=0.17$ for $1.9 \mu \mathrm{m}$ strips and $\Delta \mathrm{pCa}_{50}=0.12$ for $2.3 \mu \mathrm{m}$ strips). The $\mathrm{Ca}^{2+}$-sensitivity of force also increased as sarcomere length increased ( $\mathrm{p}=0.002$ for main effect of length), exhibiting a length-dependent activation response for both control and mavacamten-treated strips. Hill coefficients $\left(\mathrm{n}_{\mathrm{H}}\right)$ represent the degree of cooperativity in the force-pCa relationship (Fig. 4B), wherein mavacamten increased $\mathrm{n}_{\mathrm{H}}(\mathrm{p}<0.001$ for main effect of mavacamten) and 
sarcomere length did not significantly affect $\mathrm{n}_{\mathrm{H}}$.

\section{Effects of mavacamten on myocardial viscoelasticity under maximal $\mathrm{Ca}^{2+}$-activated conditions}

Sinusoidal length-perturbation analysis was used to measure the myocardium's viscoelastic characteristics and the effects of mavacamten on cross-bridge recruitment and detachment rates at maximally activated conditions (pCa 4.5). For both sarcomere lengths mavacamten reduced elastic moduli values across a wide range of frequencies (Fig. 5A-B), indicating less cross-bridge binding in mavacamten-treated strips under maximally activated conditions. These decreases in cross-bridge binding also underlie the lower force values observed for mavacamten-treated strips (Fig. 2), as changes in elastic moduli and isometric tension typically mirror each other.

Frequency shifts in the viscoelastic system response follow from changes in the enzymatic cross-bridge cycling kinetics under $\mathrm{Ca}^{2+}$-activated conditions. These frequency shifts are most easily observed in the characteristic dips and peaks of the viscous modulus vs. frequency relationship (Fig. 5C-D). Negative viscous moduli represent frequencies where the muscle produces work, and positive viscous moduli represent frequencies where the muscle absorbs work. For both sarcomere lengths mavacamten decreased the magnitude of negative viscous moduli, indicating less work production in mavacamten-treated strips than control strips. In addition, the frequencies where mavacamten-treated strips generated work was shifted towards lower frequencies. These shifts were quantified via the frequency of minimum viscous modulus for each strip $(\mathrm{p}<0.001$ for main effect of mavacamten; Fig. 6A), indicating that cross-bridge recruitment rate slowed in mavacamtentreated strips (Mulieri et al., 2002; Campbell et al., 2004). Mavacamten also increased the frequency of maximum viscous modulus ( $\mathrm{p}=0.029$ for main effect of mavacamten; Fig. $6 \mathrm{~B}$ ), indicating that cross-bridge detachment rate increased in mavacamten-treated strips at physiological temperature.

\section{Discussion}

This study contributes new biophysical observations about the effects of mavacamten on human cardiac muscle function at physiological temperature. With recent therapeutic treatments for heart failure showing promise at the sarcomere level (Teerlink et al., 2016; Heitner et al., 2019), there is a need to better define and identify molecular mechanisms of contraction that may ultimately be targeted to benefit patient care. Sarcomere length-dependent increases in $\mathrm{Ca}^{2+}$-sensitivity of contraction represent a key physiological relationship at the cellular and tissue level that underlies the Frank-Starling response in healthy hearts. Pharmaceuticals that compromised length-dependent activation may ultimately prove to be less effective therapies for heart disease (Gollapudi et al., 2017; Henze et al., 2019). Herein we show that mavacamten preserves sarcomere length-dependent increases in the $\mathrm{Ca}^{2+}$-sensitivity of force, while reducing maximum levels of force production.

These data have implications for mavacamten affecting systolic and diastolic aspects of cardiac muscle function. On the systolic side, HCM has typically been viewed as a hypercontractile phenotype due to a 'gain of function' mutation (Moore et al., 2012; Green et al., 2016). Thus, reducing the $\mathrm{Ca}^{2+}$-sensitivity of the myocardium with mavacamten treatment could reduce the hypercontractile state during systole, normalizing function and potentially leading to beneficial remodeling of the heart. Administering mavacamten (early in life) to transgenic mice carrying the myosin R403Q mutation, which typically displays a potent HCM phenotype, helped reduce ventricular hypertrophy, cardiomyocyte disarray, and myocardial fibrosis (Green et al., 2016). On the other side of a heartbeat, diastolic dysfunction leads to impaired relaxation and ventricular filling, which underlies roughly $50 \%$ of heart failure diagnoses (Aljaroudi et al., 2012; Lekavich et al., 2015; Mozaffarian et al., 2016). The findings that mavacamten reduced passive tension at both sarcomere lengths (Fig. 3B) and increased cross-bridge detachment rates under maximally activated conditions (Fig. $6 \mathrm{~B})$ suggests that mavacamten may improve relaxation.

Prior biophysical assays have shown that mavacamten slows cross-bridge recruitment, $\mathrm{P}_{\mathrm{i}}$ release, and actinassociated ADP release, all of which underlie strong cross-bridge binding and force-generation (Green et al., 2016; Kawas et al., 2017; Rohde et al., 2018). In skinned myocardial mechanics and intact myocyte assays mavacamten reduced contractility in a dose-dependent manner, slowing the rate of force development 
(Mamidi et al., 2018) and increasing the relaxation rate (Toepfer et al., 2019b, 2019a). Our frequencydependent viscoelastic stiffness data support these findings in human myocardium at physiological temperature (Fig. 5). Mavacamten shifted the negative viscous moduli-frequency relationships towards lower frequencies and reduced the amount of work generated by the myocardiaum (Fig. 5C-D), consistent with impaired force generation (Fig. 2) and slowed cross-bridge recruitment (Fig 6A). The frequency range where negative viscous moduli occur reflects the physiological range of heart rates within a species, and the diminished range for mavacamten-treated strips could indicate that mavacamten plays a limiting role at the upper end of this range. Our findings that mavacamten speeds cross-bridge detachment (Fig. 6B) agrees with data showing that mavacamten increases relaxation rates in human myofibrils (Scellini et al., 2020). Similar measurements by Scellini et al. show that mavacamten had no effect on relaxation rate in rabbit skeletal myofibrils, which suggests that the effects of mavacamten may vary with species and muscle type (Scellini et al., 2020). Additional studies will be required to more fully define the potential impacts of mavacamten on different myosin isoforms.

Previously, we used mathematical models to test how mechanosensitive contributions of the myosin OFFON equilibrium influence length-dependent activation of contraction (Campbell et al. 2018). These data showed that elevated force levels, from passive and/or active mechanisms, amplify cross-bridge recruitment and force production, more fully illustrating the fascinating (yet complicated) impacts of dynamic filament regulation (Fig. 1) that underly force production and relaxation throughout a heartbeat. Knowing that mavacamten stabilizes the OFF state (Anderson et al., 2018; Rohde et al., 2018), we wondered if sarcomere length-dependent increases in $\mathrm{Ca}^{2+}$-sensitivity would be amplified in mavacamten treated strips. Our data do not show this response in a statistically significant manner (Fig. 4A), although the pCa 50 change between $1.9 \mu \mathrm{m}$ and $2.3 \mu \mathrm{m}$ was 3 -fold greater for mavacamten treated strips (on average $\Delta \mathrm{pCa}_{50}=0.03$ for control vs. 0.09 for mavacamten-treated strips). These findings differ from a preliminary report using permeabilized myocardial strips from pigs, where mavacamten suppressed length-dependent increases from OFF-to-ON and abolished length-dependent increases in $\mathrm{Ca}^{2+}$-sensitivity of contraction (Henze et al., 2019). The functional effects of the OFF-ON equilibrium are only starting to come to light for the muscle physiology research field, yet this equilibrium represents a critical mechanism underlying regulatory coupling between the thick- and thin-filaments and the Frank-Starling law of the heart. One interesting aspect of this OFF-ON relationship is the force-dependent recruitment of myosin from the OFF-to-ON state, which encompasses a force-feedback effect to amplify contractility as force develops during isometric contractions and when muscles are stretched (Ait-Mou et al., 2016; Campbell, 2017; Fusi et al., 2017; Reconditi et al., 2017; Zhang et al., 2017; Piazzesi et al., 2018; Brunello et al., 2020). Thus, any means of modulating length-dependent changes $\mathrm{Ca}^{2+}$-sensitivity leads to significant functional effects, because myofilaments are highly cooperative and subtle shifts in pCa50 can produce large change in force-production as muscle length changes. Prior to the discovery of forcedependent OFF-ON transitions, length-dependent changes in $\mathrm{Ca}^{2+}$-sensitivity were typically thought to reflect changes in thin filament function and/or the binding kinetics of individual myosin heads (Gordon et al., 2000). Therefore, ongoing and future research testing the effects of mavacamten to preserve or even amplify length-dependent activation in transgenic models of heart failure and human tissue samples from heart failure patients will be helpful for informing the potential scope of mavacamten as a therapeutic treatment for cardiac dysfunction. More generally, these findings also imply that the OFF-ON equilibrium of myosin may be a particularly important therapeutic target for modulating myocardial function.

\section{A $\chi \varkappa \nu \circ \omega \lambda \varepsilon \delta \gamma \varepsilon \mu \varepsilon \nu \tau \sigma$}

This work was supported by the American Heart Association [17SDG33370153 to BCWT, 19TPA34860008 to BCWT and KSC, and GRNT25460003 to KSC]; the National Science Foundation [1656450 to BCWT]; and the National Institutes of Health [TR001988, HL144664, and HL133358 to KSC].

\section{Author contributions}

POA, YB, MW, and BCWT performed the research; POA, MG, KSC, and BCWT designed the research study; POA, YB, MW, MG, KSC, and BCWT carried out the data and statistical analysis; POA, MG, $\mathrm{KSC}$, and BCWT helped interpret data and potential clinical significance; POA, KSC, and BCWT wrote 
the paper.

\section{Conflict of interest}

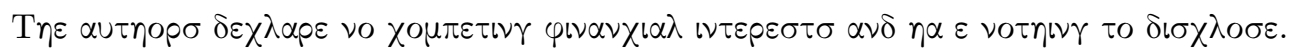

\section{References}

Ait-Mou, Y., Hsu, K., Farman, G.P., Kumar, M., Greaser, M.L., Irving, T.C., et al. (2016). Titin strain contributes to the Frank-Starling law of the heart by structural rearrangements of both thin- and thickfilament proteins. Proc Natl Acad Sci U S A 113 : 2306-2311.

Aljaroudi, W., Alraies, M.C., Halley, C., Rodriguez, L., Grimm, R.A., Thomas, J.D., et al. (2012). Impact of progression of diastolic dysfunction on mortality in patients with normal ejection fraction. Circulation $125: 782-788$.

Ambrosy, A.P., Fonarow, G.C., Butler, J., Chioncel, O., Greene, S.J., Vaduganathan, M., et al. (2014). The global health and economic burden of hospitalizations for heart failure: lessons learned from hospitalized heart failure registries. J. Am. Coll. Cardiol. 63 : 1123-1133.

Anderson, R.L., Trivedi, D. V, Sarkar, S.S., Henze, M., Ma, W., Gong, H., et al. (2018). Deciphering the super relaxed state of human beta-cardiac myosin and the mode of action of mavacamten from myosin molecules to muscle fibers. Proc. Natl. Acad. Sci. U. S. A. 115 : E8143-E8152.

Blair, C.A., Haynes, P., Campbell, S.G., Chung, C., Mitov, M.I., Dennis, D., et al. (2016). A Protocol for Collecting Human Cardiac Tissue for Research. VAD J. J. Mech. Assist. Circ. Hear. Fail. 2 :.

Brandt, P.W., Lopez, E., Reuben, J.P., and Grundfest, H. (1967). The relationship between myofilament packing density and sarcomere length in frog striated muscle. J Cell Biol 33 : 255-263.

Brunello, E., Fusi, L., Ghisleni, A., Park-Holohan, S.J., Ovejero, J.G., Narayanan, T., et al. (2020). Myosin filament-based regulation of the dynamics of contraction in heart muscle. Proc. Natl. Acad. Sci. U. S. A.117 : 8177-8186.

Campbell, K.B., Chandra, M., Kirkpatrick, R.D., Slinker, B.K., and Hunter, W.C. (2004). Interpreting cardiac muscle force-length dynamics using a novel functional model. Am J Physiol Hear. Circ Physiol.286 : H1535-H1545.

Campbell, K.S. (2017). Super-relaxation helps muscles work more efficiently. J. Physiol. 595 : 1007-1008.

Campbell, K.S., Janssen, P.M.L., and Campbell, S.G. (2018). Force-Dependent Recruitment from the Myosin Off State Contributes to Length-Dependent Activation. Biophys. J. 115 : 543-553.

Cleland, J.G., Teerlink, J.R., Senior, R., Nifontov, E.M., Mc Murray, J.J., Lang, C.C., et al. (2011). The effects of the cardiac myosin activator, omecamtiv mecarbil, on cardiac function in systolic heart failure: a double-blind, placebo-controlled, crossover, dose-ranging phase 2 trial. Lancet 378 : 676-683.

Fusi, L., Brunello, E., Yan, Z., and Irving, M. (2016). Thick filament mechano-sensing is a calciumindependent regulatory mechanism in skeletal muscle. Nat. Commun. 7 : 13281.

Fusi, L., Percario, V., Brunello, E., Caremani, M., Bianco, P., Powers, J.D., et al. (2017). Minimum number of myosin motors accounting for shortening velocity under zero load in skeletal muscle. J. Physiol.595 : $1127-1142$.

Godt, R.E., and Lindley, B.D. (1982). Influence of temperature upon contractile activation and isometric force production in mechanically skinned muscle fibers of the frog. J Gen Physiol 80 : 279-297.

Gollapudi, S.K., Reda, S.M., and Chandra, M. (2017). Omecamtiv Mecarbil Abolishes Length-Mediated Increase in Guinea Pig Cardiac Myofiber Ca2+ Sensitivity. Biophys. J. 113 : 880-888. 
Gordon, A.M., Homsher, E., and Regnier, M. (2000). Regulation of contraction in striated muscle. Physiol. Rev. $80: 853-924$.

Green, E.M., Wakimoto, H., Anderson, R.L., Evanchik, M.J., Gorham, J.M., Harrison, B.C., et al. (2016). A small-molecule inhibitor of sarcomere contractility suppresses hypertrophic cardiomyopathy in mice. Science (80-. ). $351: 617-621$.

Grillo, M.P., Erve, J.C.L., Dick, R., Driscoll, J.P., Haste, N., Markova, S., et al. (2018). In vitro and in vivo pharmacokinetic characterization of mavacamten, a first-in-class small molecule allosteric modulator of beta cardiac myosin. Xenobiotica. 1-16.

Haynes, P., Nava, K.E., Lawson, B.A., Chung, C.S., Mitov, M.I., Campbell, S.G., et al. (2014). Transmural heterogeneity of cellular level power output is reduced in human heart failure. J Mol Cell Cardiol 72 : 1-8.

Heitner, S.B., Jacoby, D., Lester, S.J., Owens, A., Wang, A., Zhang, D., et al. (2019). Mavacamten treatment for obstructive hypertrophic cardiomyopathy a clinical trial. Ann. Intern. Med. 170 : 741-748.

Henze, M., Ma, W., Wong, F., Gong, H., Anderson, R.L., Rio, C. del, et al. (2019). Length Dependent Activation in Porcine Cardiac Myofilaments is Modulated by Mavacamten. Circ. Res. 125 :.

Hooijman, P., Stewart, M.A., and Cooke, R. (2011). A new state of cardiac myosin with very slow ATP turnover: a potential cardioprotective mechanism in the heart. Biophys. J. 100 : 1969-1976.

Huxley, H., and Hanson, J. (1954). Changes in the Cross-striations of muscle during contraction and stretch and their structural interpretation. Nature $173: 973-976$.

Kampourakis, T., Sun, Y.-B., and Irving, M. (2016). Myosin light chain phosphorylation enhances contraction of heart muscle via structural changes in both thick and thin filaments. Proc. Natl. Acad. Sci. U. S. A. 113 : E3039-E3047.

Kawai, M., and Brandt, P. (1980). Sinusoidal analysis: a high resolution method for correlating biochemical reactions with physiological processes in activated skeletal muscles of rabbit, frog and crayfish. J Muscle Res Cell Motil $1: 279-303$.

Kawas, R.F.F., Anderson, R.L., Ingle, S.R.B.R.B., Song, Y., Sran, A.S.S., Rodriguez, H.M.M., et al. (2017). A small-molecule modulator of cardiac myosin acts on multiple stages of the myosin chemomechanical cycle. J. Biol. Chem. 292 : 16571-16577.

Klein, M.D., Lane, F.J., and Gorlin, R. (1965). Effect of left ventricular size and shape upon the hemodynamics of subaortic stenosis. Am. J. Cardiol. 15 : 773-81.

Lekavich, C.L., Barksdale, D.J., Neelon, V., and Wu, J.R. (2015). Heart failure preserved ejection fraction (HFpEF): an integrated and strategic review. Heart Fail. Rev. $20: 643-653$.

Linari, M., Brunello, E., Reconditi, M., Fusi, L., Caremani, M., Narayanan, T., et al. (2015). Force generation by skeletal muscle is controlled by mechanosensing in myosin filaments. Nature 528 : 276-279.

Liu, C., Kawana, M., Song, D., Ruppel, K.M., and Spudich, J.A. (2018). Controlling load-dependent kinetics of $\beta$-cardiac myosin at the single-molecule level. Nat. Struct. Mol. Biol. 25 : 505-514.

Lymn, R.W., and Taylor, E.W. (1971). Mechanism of adenosine triphosphate hydrolysis by actomyosin. Biochemistry 10 : 4617-4624.

Malik, F.I., Hartman, J.J., Elias, K.A., Morgan, B.P., Rodriguez, H., Brejc, K., et al. (2011). Cardiac myosin activation: a potential therapeutic approach for systolic heart failure. Science (80-. ).331 : 1439-1443.

Mamidi, R., Li, J., Doh, C.Y., Verma, S., and Stelzer, J.E. (2018). Impact of the Myosin Modulator Mavacamten on Force Generation and Cross-Bridge Behavior in a Murine Model of Hypercontractility. J. Am. Heart Assoc. 7 : e009627. 
Maron, B.J., Gardin, J.M., Flack, J.M., Gidding, S.S., Kurosaki, T.T., and Bild, D.E. (1995). Prevalence of hypertrophic cardiomyopathy in a general population of young adults: Echocardiographic analysis of 4111 subjects in the CARDIA study. Circulation 92 : 785-789.

McNamara, J.W., Li, A., Lal, S., Bos, J.M., Harris, S.P., Velden, J. van der, et al. (2017). MYBPC3 mutations are associated with a reduced super-relaxed state in patients with hypertrophic cardiomyopathy. PLoS One 12 : e0180064.

McNamara, J.W., Li, A., Remedios, C.G. Dos, and Cooke, R. (2015). The role of super-relaxed myosin in skeletal and cardiac muscle. Biophys. Rev. 7: 5-14.

Moore, J.R., Leinwand, L., and Warshaw, D.M. (2012). Understanding cardiomyopathy phenotypes based on the functional impact of mutations in the myosin motor. Circ Res 111 : 375-385.

Mozaffarian, D., Benjamin, E.J., Go, A.S., Arnett, D.K., Blaha, M.J., Cushman, M., et al. (2016). Heart disease and stroke statistics-2016 update a report from the American Heart Association.

Mulieri, L.A., Barnes, W.D., Leavett, B.J., Ittleman, F., LeWinter, M.M., Alpert, N.R., et al. (2002). Alterations of myocardial dynamic stiffness implicating abnormal crossbridge function in human mitral regurgitation heart failure. Circ Res $90: 66-72$.

Palmer, B.M., Suzuki, T., Wang, Y., Barnes, W.D., Miller, M.S., and Maughan, D.W. (2007). Two-state model of acto-myosin attachment-detachment predicts C-process of sinusoidal analysis. Biophys J 93 : $760-769$.

Palmer, B.M., Wang, Y., and Miller, M.S. (2011). Distribution of myosin attachment times predicted from viscoelastic mechanics of striated muscle. J. Biomed. Biotechnol. 2011 : 592343.

Piazzesi, G., Caremani, M., Linari, M., Reconditi, M., and Lombardi, V. (2018). Thick Filament MechanoSensing in Skeletal and Cardiac Muscles: A Common Mechanism Able to Adapt the Energetic Cost of the Contraction to the Task. Front. Physiol. $9: 736$.

Reconditi, M., Caremani, M., Pinzauti, F., Powers, J.D., Narayanan, T., Stienen, G.J.M., et al. (2017). Myosin filament activation in the heart is tuned to the mechanical task. Proc. Natl. Acad. Sci. U. S. A.114 : 3240-3245.

Rohde, J.A., Roopnarine, O., Thomas, D.D., Muretta, J., and Hall, J. (2018). Mavacamten stabilizes an autoinhibited state of two-headed cardiac myosin. Proc. Natl. Acad. Sci. U. S. A. 115 : E7486-E7494.

Scellini, B., Piroddi, N., Dente, M., Ferrantini, C., Coppini, R., Poggesi, C., et al. (2020). Impact of Mavacamten on Force Generation in Single Myofibrils from Rabbit Psoas and Human Cardiac Muscle. Biophys. J. 118 : 7 a.

Semsarian, C., Ingles, J., Maron, M.S., and Maron, B.J. (2015). New perspectives on the prevalence of hypertrophic cardiomyopathy. J. Am. Coll. Cardiol. 65 : 1249-1254.

Spudich, J.A. (2015). The myosin mesa and a possible unifying hypothesis for the molecular basis of human hypertrophic cardiomyopathy. Biochem. Soc. Trans. $43: 64-72$.

Stern, J.A., Markova, S., Ueda, Y., Kim, J.B., Pascoe, P.J., Evanchik, M.J., et al. (2016). A Small Molecule Inhibitor of Sarcomere Contractility Acutely Relieves Left Ventricular Outflow Tract Obstruction in Feline Hypertrophic Cardiomyopathy. PLoS One 11 : e0168407.

Stewart, S., Mason, D.T., and Braunwald, E. (1968). Impaired rate of left ventricular filling in idiopathic hypertrophic subaortic stenosis and valvular aortic stenosis. Circulation $37: 8-14$.

Teerlink, J.R., Felker, G.M., McMurray, J.J. V, Solomon, S.D., Adams, K.F., Cleland, J.G.F., et al. (2016). Chronic Oral Study of Myosin Activation to Increase Contractility in Heart Failure (COSMIC-HF): a phase 2, pharmacokinetic, randomised, placebo-controlled trial. Lancet388 : 2895-2903. 
Toepfer, C.N., Sharma, A., Cicconet, M., Garfinkel, A.C., Mücke, M., Neyazi, M., et al. (2019a). SarcTrack. Circ. Res. 124 : 1172-1183.

Toepfer, C.N., Wakimoto, H., Garfinkel, A.C., McDonough, B., Liao, D., Jiang, J., et al. (2019b). Hypertrophic cardiomyopathy mutations in MYBPC3 dysregulate myosin. Sci. Transl. Med. 11 :.

Tuohy, C.V., Kaul, S., Song, H.K., Nazer, B., and Heitner, S.B. (2020). Hypertrophic cardiomyopathy: the future of treatment. Eur. J. Heart Fail. 22 : 228-240.

Wilson, W.S., Criley, J.M., and Ross, R.S. (1967). Dynamics of left ventricular emptying in hypertrophic subaortic stenosis. A cineangiographic and hemodynamic study. Am. Heart J. 73 : 4-16.

Zhang, X., Kampourakis, T., Yan, Z., Sevrieva, I., Irving, M., and Sun, Y.-B. (2017). Distinct contributions of the thin and thick filaments to length-dependent activation in heart muscle. Elife 6 : e24081.

\section{Figure Legends}

Figure 1: Schematic introducing dynamic filament coupling between thick- and thin-filaments. Thin-filament regulation involves $\mathrm{Ca}^{2+}$ binding to troponin and subsequent movement of tropomyosin to expose actin sites along the thin filament, to which myosin can bind and form force-generating cross-bridges. Thick-filament regulation involves myosin OFF-ON transition kinetics, which is a mechanosensitive equilibrium that shifts myosin heads from OFF to ON as muscle force increases. Myosin heads in the OFF state cannot bind actin, while those in the ON state can bind actin to form force-generating cross-bridges. This dynamic regulatory coupling implies that any modification to thin-filament function will in turn change the status of thick-filament regulation, and vice versa (figure adapted from (Campbell et al., 2018) ).

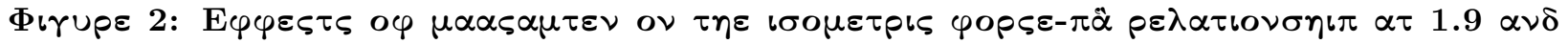

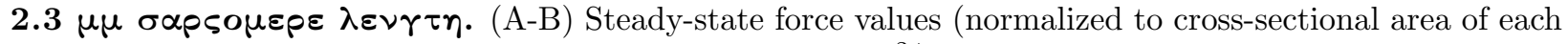
myocardial strip) are plotted against $\mathrm{pCa}\left(\mathrm{pCa}=-\log _{10}\left[\mathrm{Ca}^{2+}\right]\right)$. Lines represent 4-parameter Hill fits to Eq. 1. Dashed lines show fits at $1.9 \mu \mathrm{m}$ sarcomere length, replotted in panel B. Data were gathered from 6 hearts, with a total of 17 control strips and 18 mavacamten strips at $1.9 \mu \mathrm{m}$ sarcomere length, and 18 control strips and 17 mavacamten strips at $2.3 \mu \mathrm{m}$ sarcomere length. Data shown as mean \pm SEM, error bars within symbol if not visible.

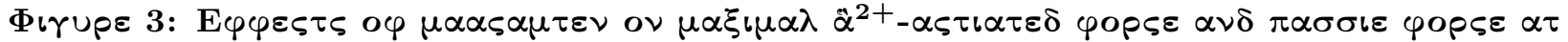

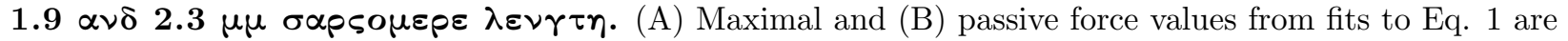
shown for each myocardial strip from each experimental group. Significant main effects and the associated interaction from linear mixed models analysis are listed above each panel for respective data therein. Jitter plots (colored symbols) show measurements for each myocardial strip, with n listed in the legend of Fig. 2. Black symbols show mean \pm SEM for each group plotted to the left of individual measurements.

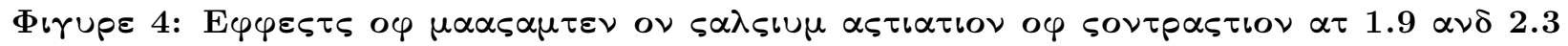

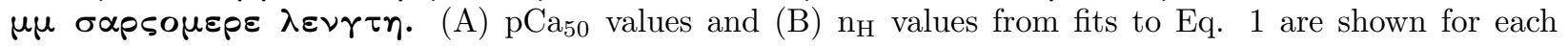
myocardial strip from each experimental group. Significant main effects and the associated interaction from linear mixed models analysis are listed above each panel for respective data therein. Jitter plots (colored symbols) show measurements for each myocardial strip, with $n$ listed in the legend of Fig. 2. Black symbols show mean \pm SEM for each group plotted to the left of individual measurements.

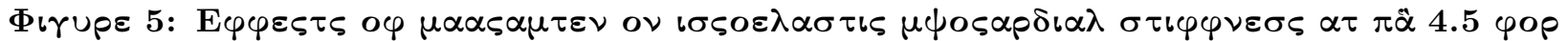

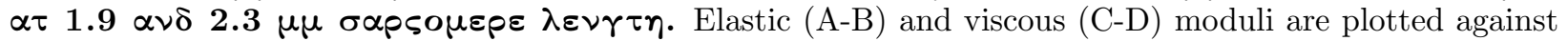
frequency for maximal $\mathrm{Ca}^{2+}$-activated conditions. Data shown as mean $\pm \mathrm{SEM}$, with $\mathrm{n}$ listed in the legend of Fig. 2.

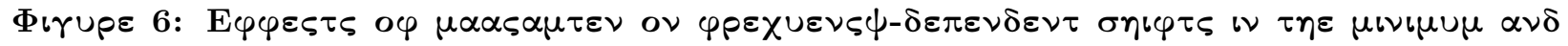

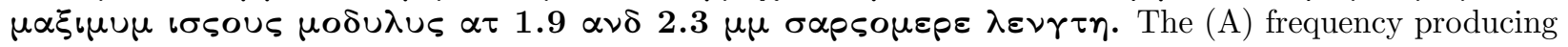
the minimum viscous modulus and (B) frequency producing the minimum viscous modulus from polynomial fits to these associated regions of interest. Frequency shifts in the minimum and maximum viscous modulus 
describe relative changes in cross-bridge recruitment and detachment rates, respectively. Significant main effects and the associated interaction from linear mixed models analysis are listed above each panel for respective data therein. Jitter plots (colored symbols) show measurements for each myocardial strip, with $\mathrm{n}$ listed in the legend of Fig. 2. Black symbols show mean \pm SEM for each group plotted to the left of individual measurements.

\section{Tables}

Table 1: Organ donor characteristics.

\begin{tabular}{llllll}
\hline Sample ID & Age (yr) & Sex & Race & Diabetic & Cause of Death \\
\hline FC3CB & 10 & F & White $^{*}$ & No & Anaphylaxis, anoxia \\
24713 & 47 & F & White $^{*}$ & No & Head trauma \\
BE497 & 56 & M & Black $^{*}$ & No data & Cardiac arrest \\
31331 & 58 & F & White $^{*}$ & No & Anoxia \\
CF462 & 61 & F & White $^{*}$ & Yes & Stroke \\
2508D & 41 & M & White & No data & Stroke \\
\hline
\end{tabular}

*Not Hispanic or Latino

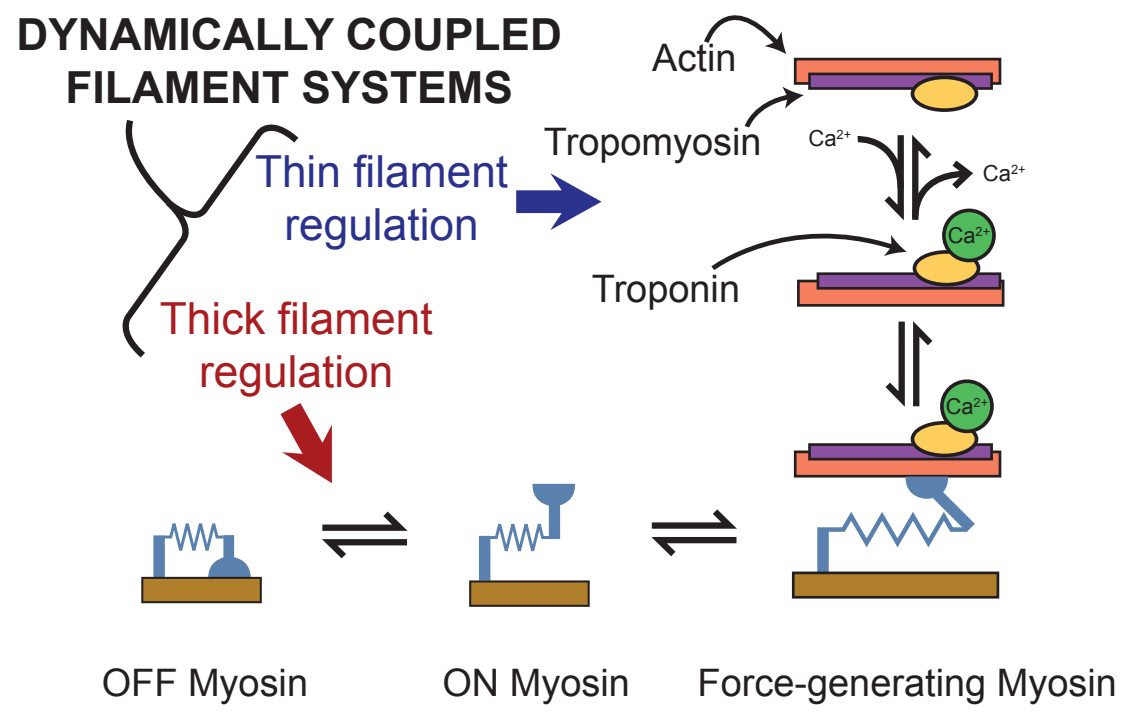



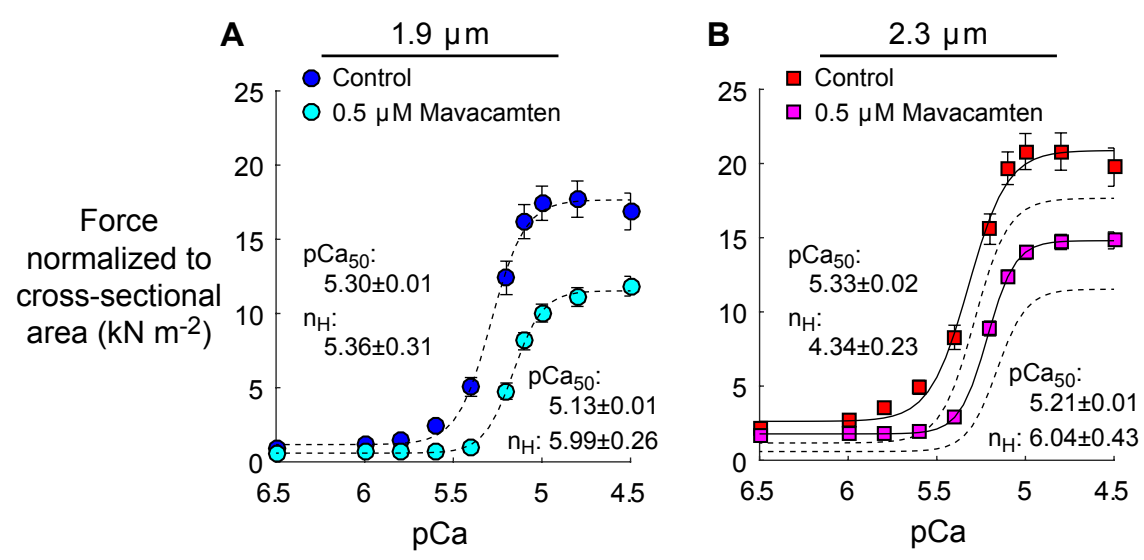
A

Mavacamten, $p<0.001$

Length, $p=0.005$

Mavacamten*Length, $p=0.854$

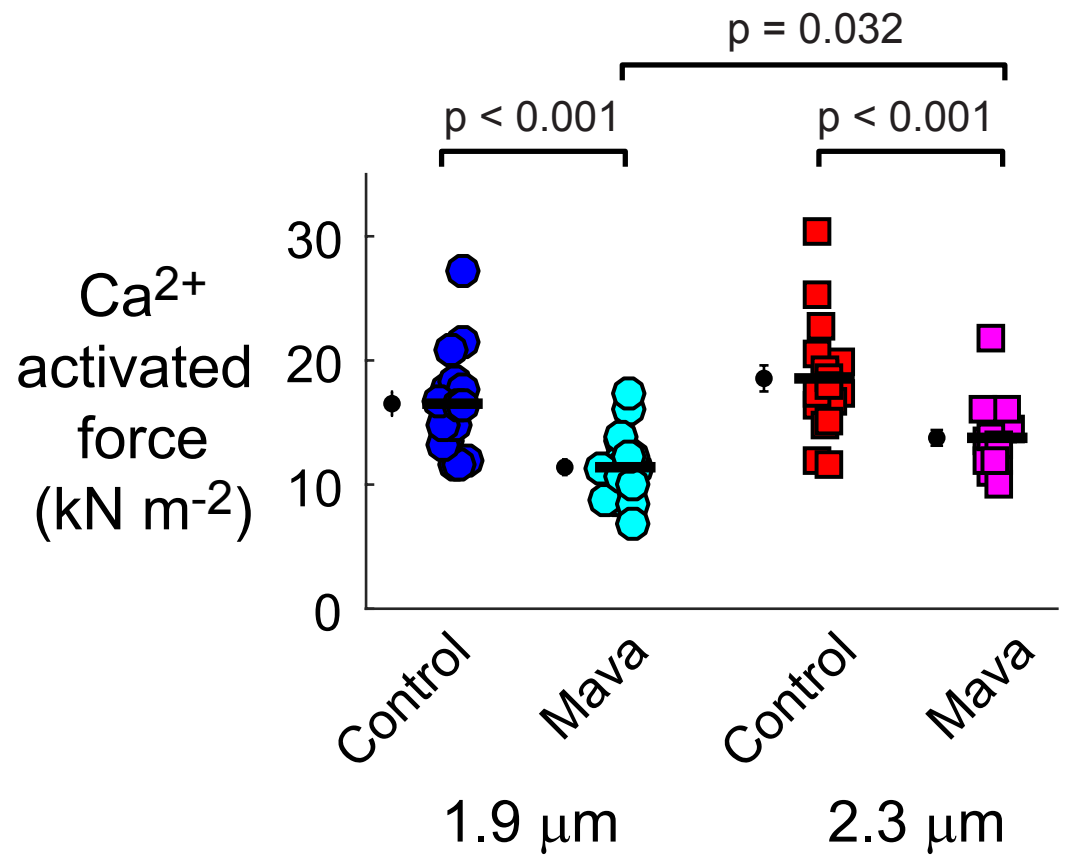

B

Mavacamten, $p<0.001$

Length, $p<0.001$

Mavacamten*Length, $p=0.264$

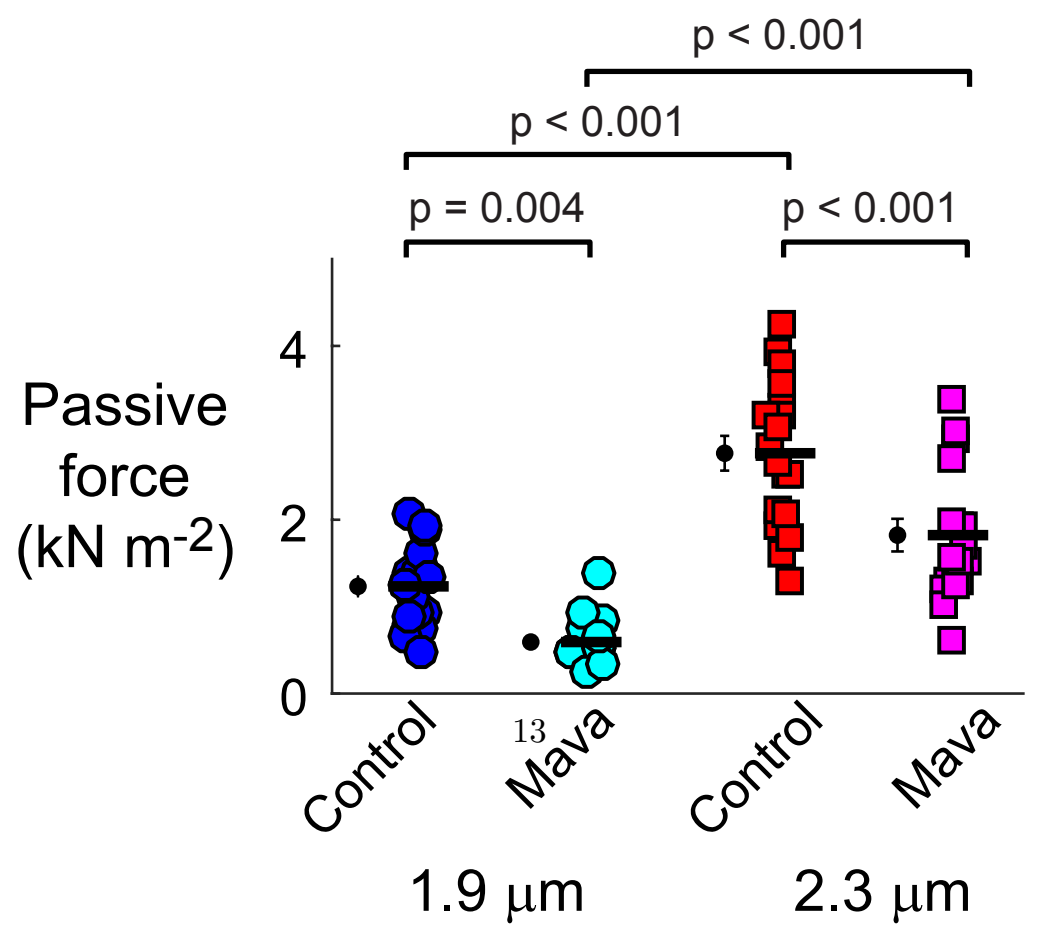


A

Mavacamten, $p<0.001$

Length, $p=0.002$

Mavacamten ${ }^{*}$ Length, $p=0.797$

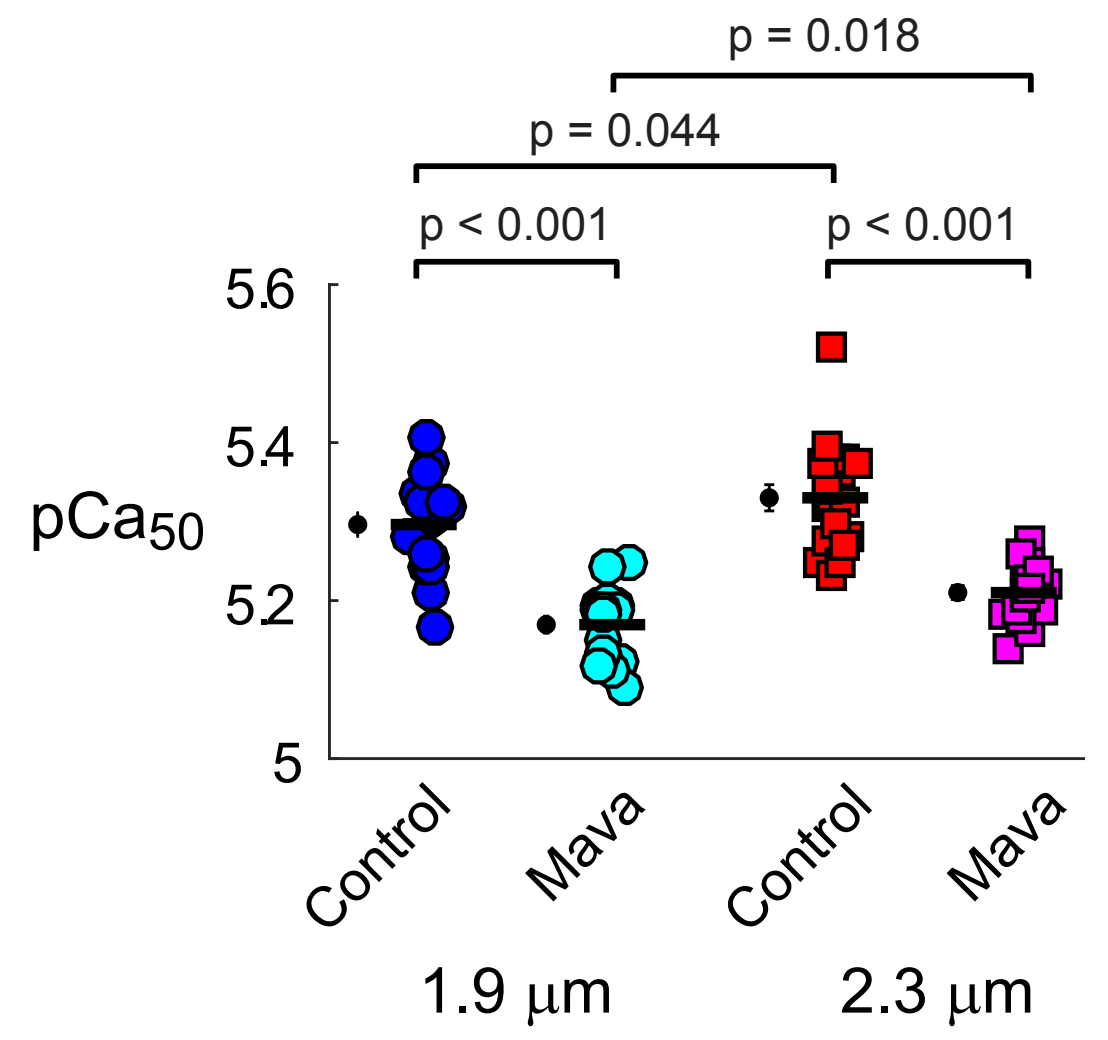

B Mavacamten, $p<0.001$

Length, $p=0.098$

Mavacamten*Length, $p=0.076$

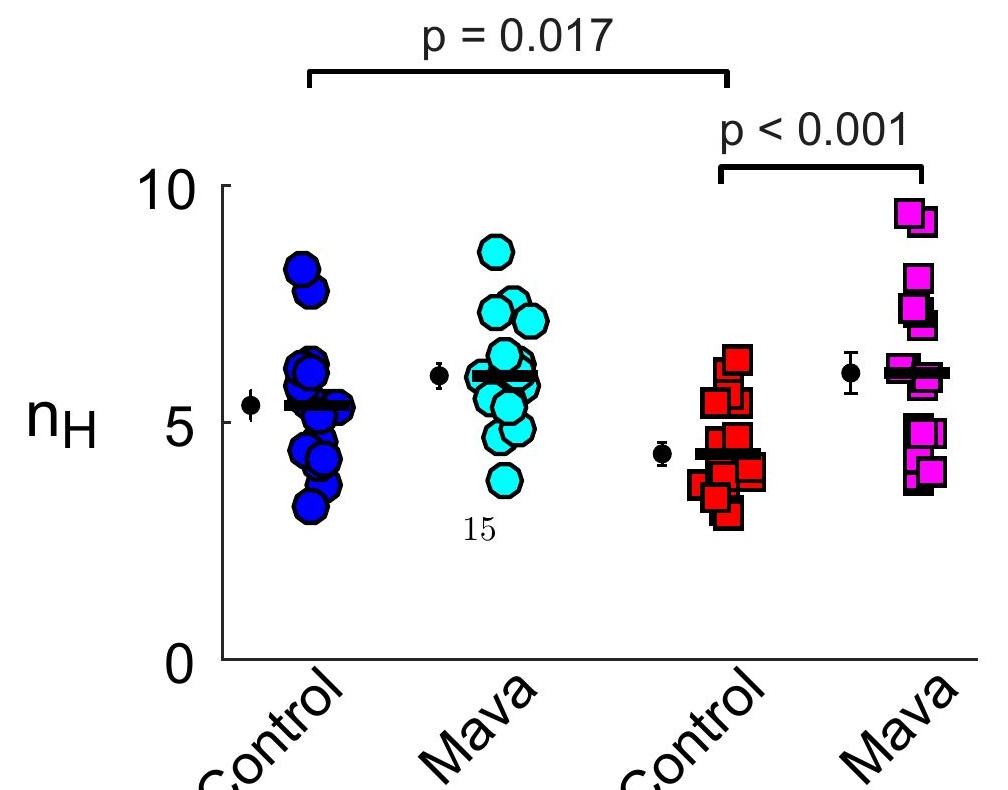



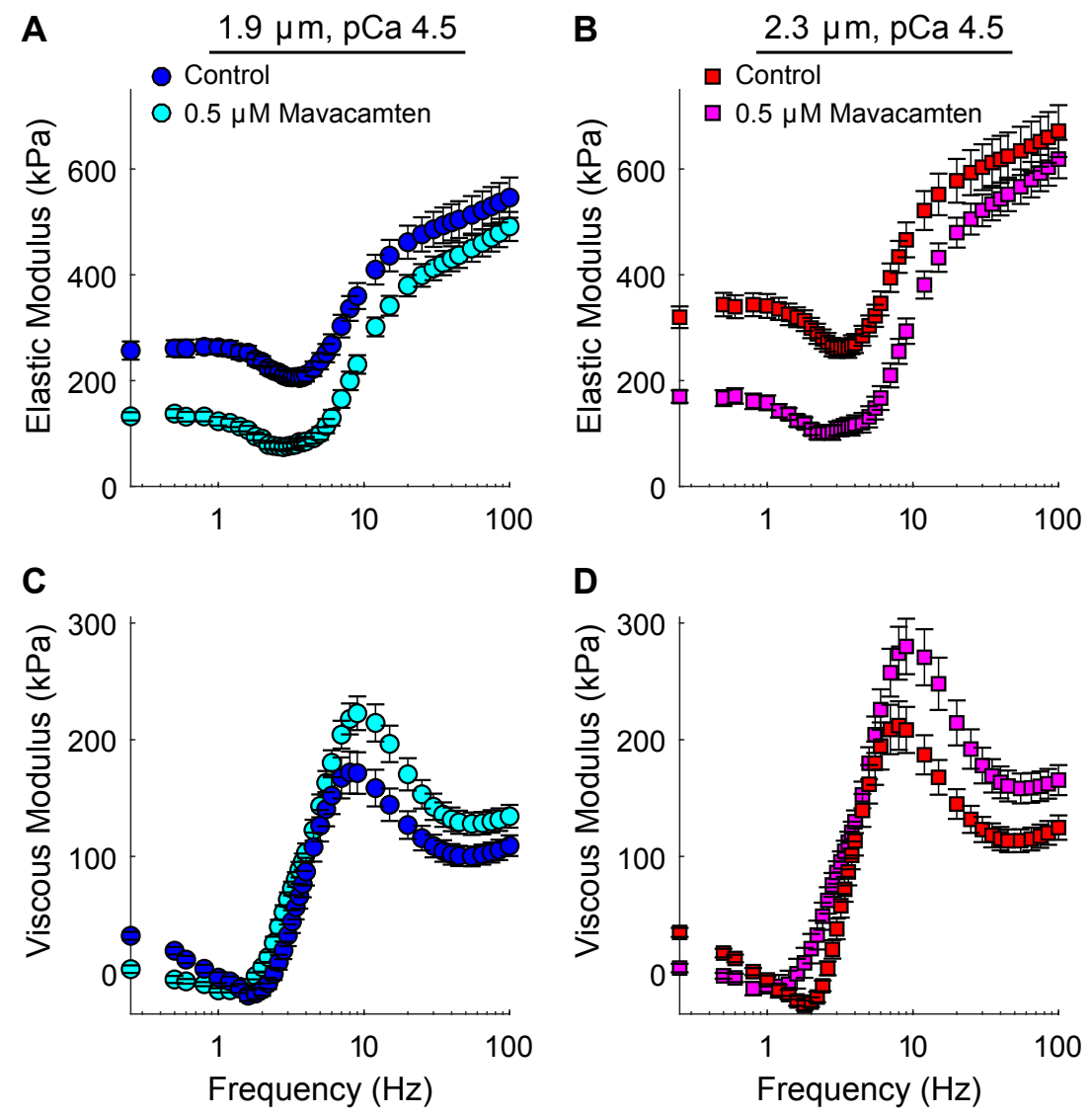
Mavacamten, $p<0.001$

Length, $p=0.344$

Mavacamten*Length, $p=0.725$

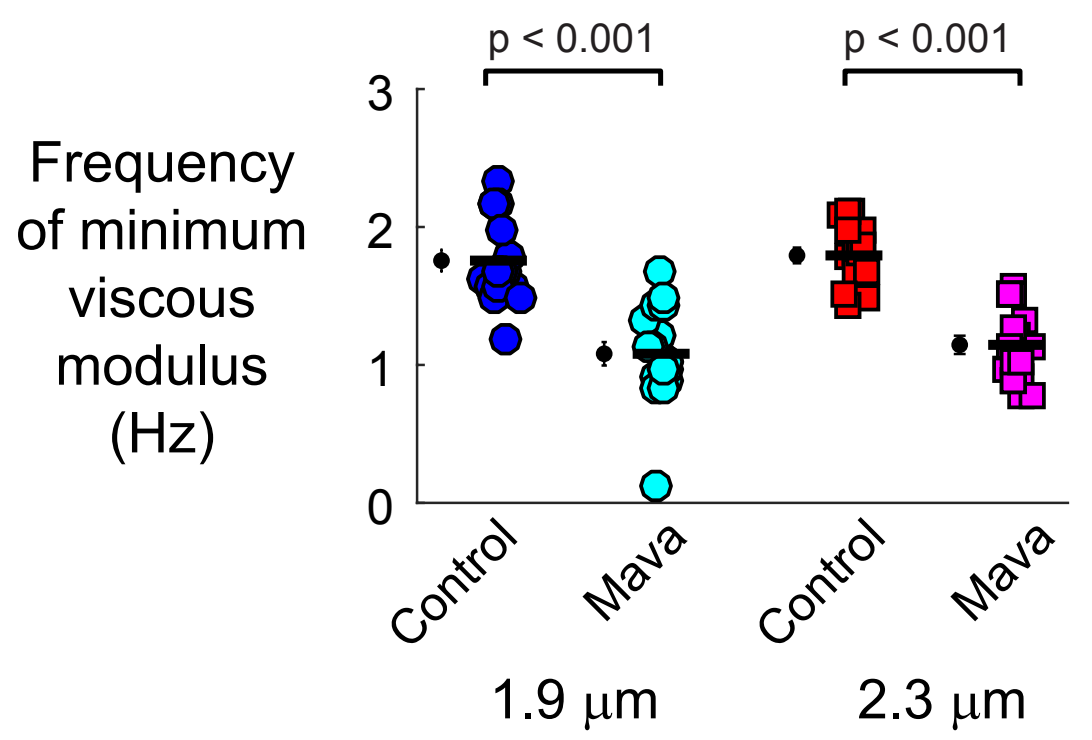

B

Mavacamten, $p=0.029$

Length, $p=0.766$

Mavacamten*Length, $p=0.854$

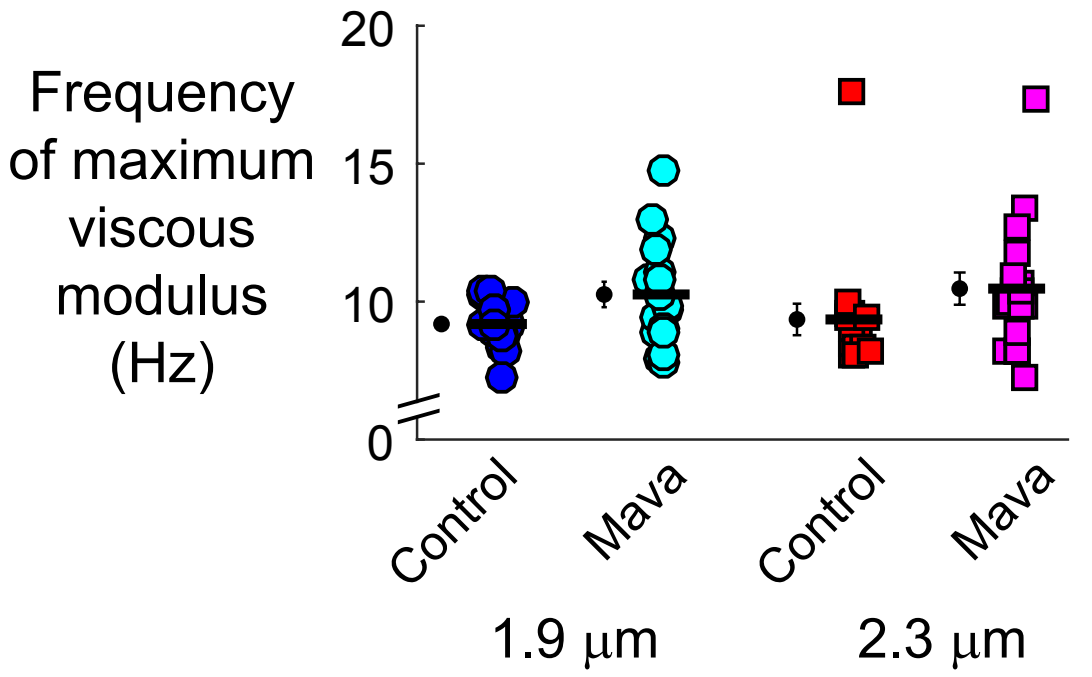

